\title{
Sexualidad y redes sociales online: Una experiencia educativa con adolescentes de Montevideo
}

\section{Sexuality and online social networks: An educational experience with adolescents from Montevideo}

\author{
Gonzalo Iván Gelpi ${ }^{1}$ (1) @; Nutarel Pascoll ${ }^{1}$ 이 @; Dasha Egorov ${ }^{1}$ (1) @ \\ ${ }^{1}$ Universidad de la República (UdelaR), Uruguay
}

\begin{abstract}
Resumen. El artículo da cuenta de una experiencia de ciclo de talleres con aproximadamente 300 adolescentes de instituciones públicas de Enseñanza Media de la ciudad de Montevideo (Uruguay). Las actividades se ejecutaron durante el segundo semestre del 2017 en el marco de un convenio entre el Programa Género, Salud Reproductiva y Sexualidades de la Facultad de Psicología (UdelaR) y el Consejo de Educación Secundaria. Además, se contó con el apoyo del Programa de Educación Sexual de la Administración Nacional de Educación Pública. Los objetivos más importantes del ciclo eran: reflexionar en torno a las prácticas cotidianas de interacción social de los adolescentes en las redes sociales online; debatir sobre la producción y difusión de contenidos de índole sexual en las redes sociales online e intercambiar conocimiento sobre prácticas sexuales novedosas en dichas redes, visualizando los riesgos potenciales. El análisis cualitativo-interpretativo propuesto se basa en los discursos de los participantes en función de las técnicas aplicadas en los talleres. A partir de la información obtenida se definieron categorías analíticas operativas a desarrollar en el presente trabajo.
\end{abstract}

Palabras clave: adolescentes; enseñanza media; sexualidad; TIC; redes sociales

Abstract. This paper discusses the experience of workshops made with approximately 300 adolescents from public high schools in Montevideo (Uruguay). The activities were held during the second half of 2017 within the framework of an agreement between the Gender, Reproductive Health and Sexualities Program of the Faculty of Psychology (UdelaR) and the Secondary Education Council. It also had the support of the Sexual Education Program of the National Administration of Public Education. The most important objectives were: to reflect on the daily practices of social interaction of adolescents in online social networks; discuss the production and spread of sexual content, and exchange knowledge about sexual practices in these networks, visualizing the potential risks. The proposed qualitative-interpretative analysis is based on the discourses of the participants based on the techniques applied in the workshops. Based on the information obtained, it was possible to define the operational analytical categories elaborated in the present work.

Keywords: adolescents; high school; sexuality; ICT; social networks

\section{Introducción}

\subsection{La expansión de las TIC y la era de las redes sociales online}

A partir de los años 70 la globalización en todas sus dimensiones facilitó que se produjera una nueva revolución tecnológica. Los avances se gestaron paulatinamente, pero con el advenimiento del nuevo milenio se asistió a una aceleración inusitada en la historia de la humanidad en lo que refiere a la posibilidad de conexión y acceso a la información. Desde finales de los años 80, para algunos investigadores del área sociales, es una preocupación indagar sobre el impacto de las nuevas tecnologías en los procesos de socialización e interacción (Turkle, 1984; Warren, 1990; Smith, 1992; Dean, 1994; Somers, 1994; Fox, 1998; Turkle, 1999; Kendall, 2000; Stryker y Burke, 2000; Rudolph, 2003). En la actualidad es difícil medir el 
impacto del fenómeno porque constantemente se producen transformaciones, creaciones e innovaciones que afectan el modo de ser, estar y sentir de los sujetos. Las Tecnologías de la Información y la Comunicación (TIC) se desperdigaron masivamente en todo el planeta, forman parte de la vida cotidiana y para la mayoría de la población occidental son indispensables porque facilitan diversas actividades (Fernández Sánchez, 2013; Linne, 2018).

En Uruguay, en los últimos años, el acceso y uso de internet ha crecido significativamente. La calidad y rapidez de conexión es de las mejores del mundo. El $80 \%$ de la población uruguaya utiliza internet (Grupo Radar, 2016). Un estudio revela que cada vez es menor la "brecha digital" entre los distintos segmentos de la población y casi dos tercios de los hogares tienen wifi (Grupo Radar, 2016). Entre los muchos usos posibles de internet, en la población uruguaya se destacan: buscar información, recrearse (ya sea mediante juegos, escuchar música u otros) y, principalmente comunicarse (Noboa, 2010). El aumento del uso de smartphones en el país diversificó los lugares de conexión y amplió el tiempo que se dedica a estar en línea (Márquez, 2017). Las redes sociales online y el chat son los usos más frecuentes de los usuarios (Grupo Radar, 2016). Con respecto a las redes sociales online es importante destacar que, las mismas "no deben entenderse como simples herramientas tecnológicas para el intercambio de mensajes, si en algún momento de su corta historia lo fueron, sino como auténticos medios para la comunicación, la interacción y la participación global" (Del Hoyo, Fernández y García, 2014, p.36).

En este contexto, "las relaciones entre los seres humanos tienen un nuevo espacio donde generarse, nuevos espacios de intercambios informativos" (Cornejo y Tapia, 2011, p.221). Como presenta Bernete (2009), ahora es posible conocer personas, comunicarse, comprar, divertirse y producir a través de internet. Estas nuevas formas de comunicarse y producir no solo modifican y moldean comportamientos cotidianos de los sujetos, sino que también se modifica la propia forma del sujeto de ver al mundo (Topa, 2010). Mediante las TIC se asiste a novedosas modalidades de producción de subjetividad y configuraciones identitarias. La identidad digital amplía la identidad pública de las personas, dándole nuevas formas de ser, rehacerse y mostrarse. Esta identidad digital comprende todo lo que el sujeto realiza a través de internet, desde lo más público, como las publicaciones que tienen como objetivo ser vistas por el resto de la comunidad virtual en las redes sociales online (posteos, fotos, videos, transmisiones en vivo y mensajes que expresen emociones y/o sentimientos de diversa índole) hasta aspectos más privados, donde los usuarios no tienen intención de que sean de orden público (como puede ser el historial de búsqueda de internet, entre tantos otros) (Sabater, 2014). 
Los sujetos participan activamente en la creación de su perfil público digital, los mismos intentan que se visualice en las redes sociales online lo que quieren dar a conocer de sí mismos, mayoritariamente esto se da en función de las expectativas deseadas en cada grupo social de referencia y/o pertenencia, pero teniendo en consideración elementos subjetivos. Como presenta Sabater (2014), "las TIC representan el soporte de esta exhibición de contenidos asociados a la vida privada" (p.3). Pero esta exhibición no es una muestra objetiva de la realidad de la persona. Los usuarios eligen el material privado de su vida que exponen y a quiénes la exponen, armando así una versión determinada de sí mismo en las redes. "Los sujetos utilizan todos los recursos que tienen a mano y administran las estrategias con las que cuentan para el armado de sus propias narrativas personales, para ficciones de realidad" (Narvaja y De Piero, 2016, p.242). En ocasiones, la construcción de la identidad digital genera consecuencias impensadas, dado que, no siempre se hace público lo que los sujetos deseaban, ni todos los contenidos que se producen acaban estando exclusivamente bajo control de los usuarios. Por esa razón éstos defienden el derecho "al olvido" por considerar que algunos contenidos disponibles en la web pueden perjudicar su reputación (Sibilia, 2018a). Lo público y lo privado se ha resignificado en el último tiempo. Sibilia (2009) expresa que hay un cambio en la formación subjetiva a partir de las nuevas tecnologías, la subjetividad se ha «exteriorizado». "El nuevo modelo es, antes que nada, un tipo subjetivo que desea ser amado y deseado, que busca desesperadamente la aprobación ajena y, para lograrlo, intenta tejer contactos y relaciones íntimas con los demás" (Sibilia, 2009, p. 312).

Las redes sociales online pasan a ser uno de los ámbitos fundamentales para la realización de los sujetos porque necesitan del reconocimiento de los otros (Sibilia, 2015). Las relaciones interpersonales son influenciadas por el acceso cotidiano a internet y por el uso diario de las redes y de los chats. Como presentan Cornejo y Tapia (2011), a las relaciones caracterizadas por el cara a cara, se les suman las relaciones virtuales, las cuales se caracterizan por la aceleración en los procesos vinculares. Esta nueva forma de conexión y de relación virtual, hace posible que lo que antes pertenecía al ámbito privado y familiar, ahora se expone de forma pública en las redes sociales online. Este fenómeno es llamado extimidad, este engloba las conductas de exhibición y los deseos o necesidad de hacerlo (Narvaja y De Piero, 2016). Desde la perspectiva de Sibilia (2008) la extimidad "consiste en exponer la propia intimidad en las vitrinas globales de la red" (p.16). 


\subsection{Los adolescentes contemporáneos y su relación con las TIC}

Según Klein (2004) la adolescencia es una invención de la modernidad resultado de un desarrollo de procesos sociales, económicos y políticos. Esto hace de la adolescencia una etapa y una categoría de reciente aparición en la historia de la humanidad, lo que se torna un desafío incluso epistémico en tanto campo de estudios, eje de políticas y categoría social (Ramos, 2015).

La etapa de la adolescencia presenta cambios en los sujetos, no solo biológicos y fisiológicos, sino también psicológicos. Viñar (2009) afirma que lo más pertinente, es hablar de adolescencias en plural a causa de los diversos modos de vivir, sentir y a veces de padecer de los adolescentes. Las características de cada adolescente "son producto de un cúmulo de interacciones entre lo individual y lo social en donde las categorías sexo, género, edad, nivel socioeconómico, lugar de procedencia y residencia, etnia y orientación sexual entre otros, se articulan de manera compleja" (Ramos, 2015, p.17).

Cuando se hace mención a los adolescentes uruguayos contemporáneos, es posible identificar algunas características comunes a este grupo etario: nacieron en democracia, crecieron con la globalización, la crisis de la modernidad, la revolución científica tecnológica, la era de la informática y las nuevas tecnologías, el auge de las redes sociales online, las crisis económicas y de las funciones protectoras del Estado-nación y sus instituciones, los fuertes cambios en las familias y la subjetividad, ejercen activamente su sexualidad a edades más tempranas que antiguas generaciones, muchos rechazan las etiquetas sexuales y fueron testigos de la revolución del movimiento feminista y de la diversidad sexual (Ramos, 2015).

La conformación de la identidad, la búsqueda de pares y de grupos de pertenencia son aspectos centrales en el ser adolescente (Regalado et al., 2012). Las redes sociales online son un medio propicio para comunicarse y conectarse con el grupo de pares y mantener la conexión en todo momento. Esta forma de conectar no se limita únicamente al grupo de amigos o conocidos, sino que va mucho más allá, ampliando sus interacciones con otros usuarios para compartir afinidades, intereses, crear nuevas relaciones, etc. (Regalado et al., 2012). Según Balaguer (2008) las redes sociales online se constituyen, en simultáneo con los grupos de pares, como una contención, como un apoyo, siendo un lugar de descarga para los adolescentes. Las mismas operan como "holding electrónico" por su función maternante de sostén distribuido entre todos los contactos, usuarios o "amigos". También cabe destacar que, la extimidad es un fenómeno que forma 
parte del contexto en el que se desarrollan y socializan, es por esto, entre otros motivos, que los adolescentes adhieren a dicha práctica en las redes e inclusive se fomenta socialmente.

En Uruguay, la franja etaria comprendida entre 12-19 años alcanzó un tope de penetración y popularidad en relación con el uso de internet y en particular de las redes sociales online (Grupo Radar, 2016). En la franja etaria de 15-19 años, un $92,3 \%$ del total de adolescentes tiene cuenta en Facebook, varios tienen cuentas en otras redes, especialmente en Instagram y Snapchat. Cabe resaltar que, en el país, el 60\% de adolescentes y jóvenes tiene celular con conexión a internet (MIDES- ENAJ, 2015).

\subsection{Los adolescentes y la sexualidad en la virtualidad}

La sexualidad es una de las dimensiones fundamentales de las relaciones humanas y produce identidad. "Pensar la sexualidad en clave identitaria ha configurado un particular ordenamiento por el cual las prácticas sexuales otorgan identidad" (Fernández, 2015, p.3). En la adolescencia como en otras etapas de la vida, la sexualidad cuenta con características, manifestaciones, necesidades y demandas particulares. En este periodo, la transformación corporal, el autoerotismo, el inicio de las prácticas masturbatorias, el comienzo de la capacidad reproductiva y la iniciación sexual cobran vital importancia (López Gómez, 2015). Por lo antes expuesto, es importante observar como cada persona organiza y vive, de forma consiente e inconsciente su sexualidad y cómo se construye mediante la articulación compleja de sus características sociales, económicas, culturales, raciales y de género (López Gómez, 2015).

Actualmente no hay ninguna dimensión de los sujetos que quede excluida de la transformación generada por la innovación tecnológica y las posibilidades de manejar la información, eso incluye a la sexualidad (Cornejo y Tapia, 2011). Los cambios considerados en lo público/privado también se dieron en esta materia. La sexualidad en sí misma, antes, era un asunto privado, que se hablaba en lo íntimo y con alguien de confianza (Louro, 2000). Hoy en día, la sexualidad, es un fetiche de las sociedades modernas. La misma y sus representaciones lo invaden todo, pasando a ser mercantilizada en todos los sentidos posibles (Giddens, 1992).

En un contexto donde la extimidad es base para el relacionamiento de los usuarios en las redes sociales online, la sexualidad no está exenta de su influencia. "Los estereotipos modernos de la sexualidad han entrado en acelerada mutación 
desde sus demarcaciones de lo íntimo o lo privado -como las perfomances posporno- hasta las estéticas de la seducción y la producción de las corporalidades" (Fernández, 2015, p.6). En los últimos años se han desarrollado nuevos comportamientos de índole sexual que se realizan a través de las TIC. Hoy en día, los fenómenos que se informan con mayor frecuencia son el sexting ${ }^{1}$, el cibersexo y las relaciones afectivo-sexuales virtuales, aunque también preocupa mucho la prevalencia del grooming ${ }^{2}$. Excepto el grooming, el resto no configuran en sí mismas un problema, sin embargo, en función de las acciones que realicen los sujetos podrían constituir uno (casos de sexrevenge, sextorsion, fakes profiles, entre otros). Estos comportamientos y estas nuevas prácticas sexuales no son exclusivas de los adolescentes, a pesar de que en esa etapa la tasa es elevada (Velázquez, López y Arellano, 2013).

A su vez, la literatura internacional, sostiene que, los casos de cyberbullying, sextorsion, fotos y videos de contenido erótico/sexual que se viralizan y el grooming, tienen consecuencias negativas en la esfera bio-psico-social de las víctimas, por ejemplo: deterioro de la autoestima, crisis de angustia y ansiedad, depresión, aislamiento social, intentos de autoeliminación y suicidios consumados, entre otros (Pérez et al., 2011; Mercado, Pedraza y Martínez, 2016).

A la fecha, en Uruguay, este campo de estudios ha sido poco explorado y al momento no se conocen cifras sobre los fenómenos antes mencionados. Igualmente, el Departamento de Delitos Tecnológicos de la Dirección General de Lucha Contra el Crimen Organizado del Ministerio del Interior ha manifestado su preocupación por el incremento de situaciones que involucran adolescentes y la vulneración de sus derechos humanos. Las instituciones educativas de Enseñanza Media también han mostrado preocupación por la alta prevalencia de casos de sexting, sextorsión y acoso virtual. Al tratarse de un fenómeno que ha ganado mayor notoriedad social, los referentes de sexualidad (figura institucional destinada directamente a la implementación del Programa de Educación Sexual) manifiestan la escasa capacitación para dar respuestas sólidas y eficaces a las problemáticas antes mencionadas.

\footnotetext{
${ }^{1}$ Término que se refiere al envío de mensajes sexuales, eróticos o pornográficos, por medio de teléfonos móviles u ordenadores.

2 Es una práctica de acoso a través de las redes sociales, emprendida por adultos en contra de niños y jóvenes con el objetivo de ganarse la amistad de estos, creando una conexión emocional, con el fin de disminuir las preocupaciones de los menores y poder abusar sexualmente de ellos.
} 


\section{Método}

\subsection{Contexto de la propuesta}

El ciclo de talleres sobre sexualidad en las redes sociales online para adolescentes fue en el marco de la práctica pre-profesional titulada: "Talleres de género y diversidad sexual en Enseñanza Media", la cual fue ofertada en el Ciclo de Graduación de la Licenciatura en Psicología de la Universidad de la República. Dicha propuesta surgió a partir de la realización de un proyecto de investigación sobre temas afines al género y la diversidad sexual en Enseñanza Media Superior durante el período comprendido entre los años 2016-2018. Las actividades realizadas fueron amparadas en el Convenio vigente entre el Programa Género, Salud Reproductiva y Sexualidades de la Facultad de Psicología (UdelaR) y el Consejo de Educación Secundaria (CES), además de contar con el aval del Programa de Educación Sexual (PES). Las temáticas trabajadas en los talleres derivaron de demandas institucionales de los propios centros. Cada instancia taller tuvo una duración aproximada de hora y media. Los objetivos de las actividades fueron: a) reflexionar en torno a las prácticas cotidianas de interacción social de los adolescentes en las redes sociales online; b) debatir sobre la producción y difusión de contenidos de índole sexual en las redes sociales online; c) intercambiar conocimiento sobre prácticas sexuales novedosas en las redes sociales online, visualizando los riesgos potenciales; d) hacer énfasis en incorporar en el intercambio las perspectivas de género, diversidad y derechos; y, e) difundir información específica sobre espacios donde pueden asesorarse legal y/o psicológicamente ante casos de vulneración de derechos vinculados a delitos informáticos.

Esta experiencia es pertinente para el beneficio de la comunidad educativa porque se centra en problemáticas de reciente aparición, de alta prevalencia, que interpelan al mundo adulto y a su capacidad de respuesta, por ende, este acercamiento a la realidad de los centros educativos y a los relatos de los y las adolescentes, podría ser un punto de partida para futuras intervenciones y coopera en la delimitación de nuevos temas de investigación en esta materia.

Los talleres fueron coordinados por dos estudiantes de la Licenciatura en Psicología próximos al egreso, contando con la presencia, el apoyo y la supervisión de los responsables académicos de la propuesta curricular y con el referente de sexualidad del centro correspondiente, en el caso que dicha institución contara con uno. Las actividades se desarrollaron en el segundo semestre del 2017. En total se completaron 15 talleres en instituciones educativas públicas de Enseñanza Media. La 
mayoría de ellos abordaron temáticas como la violencia en vínculos afectivo-sexuales y la sexualidad en las redes sociales online, de hecho, el último punto señalado fue transversal en todos los talleres planificados.

\subsection{Participantes}

La propuesta se ofreció a 8 instituciones públicas de Enseñanza Media de la ciudad de Montevideo. Se trabajó con instituciones localizadas en distintos barrios de la ciudad y que reciben estudiantes de diversas zonas de la capital, lo que garantizó que la población participante fuera lo más heterogénea posible. Finalmente, se logró concretar 15 talleres en 6 instituciones públicas de Enseñanza Media ubicadas en los barrios Malvín Norte, Malvín, Parque Batlle, Pocitos, Aguada y Centro.

Los talleres fueron planificados para adolescentes de los distintos centros educativos. Los mismos, se ejecutaron en horas de clase cedidas por profesores y/o horas puente. Por lo general, el espacio asignado para las actividades fue el salón de proyecciones de cada centro o, en su defecto, el salón de clase. Del ciclo de talleres aproximadamente participaron unos 300 adolescentes.

\subsection{Procedimientos}

Para que las experiencias fueran comparables entre sí, se ejecutó la misma técnica en todos los talleres específicos sobre sexualidad en las redes sociales online en los diferentes centros educativos. Al inicio de cada taller se realizaba un caldeamiento con el objetivo de generar un óptimo clima de trabajo. Éste, consistía en que los adolescentes circularan por el aula y se agruparan dependiendo de quienes tenían cuentas en alguna red social y quienes no, quienes usaban más Facebook que Instagram, quienes tenían Snapchat, cuáles redes creían que eran más populares, quienes tenían a sus padres en las redes, quienes las usaban para jugar online, para conocer gente, entre otras consignas. Luego sí se ejecutaba la técnica. La misma, consistía en la reproducción de un compilado de escenas de la película Disconnect, las cuales se centran en la situación vivida por Ben Boyd, el protagonista. El objetivo de la técnica era sensibilizar a los adolescentes sobre los efectos que produce un uso irresponsable de las redes sociales online y abrir el debate en torno a las prácticas sexuales virtuales y cómo se produce sexualidad en las redes sociales online. En ese sentido, las opiniones, creencias y experiencias relatadas de los estudiantes se articulaban con conceptos teóricos y de ese modo se propiciaba una co-construcción de saberes. 
Luego del intercambio, se les pedía a los estudiantes que se reunieran en equipos de a 5 personas para que escribieran en una hoja A4 los riesgos y las precauciones a tener en cuenta en torno a la práctica del sexting y del cibersexo. El objetivo de la técnica consistía en informar y debatir con los adolescentes los riesgos que acarrea el mal uso de las redes sociales online, de ese modo, se pretendía promover prácticas seguras y brindar información sobre las redes de apoyo existentes.

La información obtenida durante las actividades desarrolladas se documentó en diarios de campo realizados por los talleristas y los coordinadores académicos de la propuesta. En las bitácoras se registraron las observaciones vinculadas al lenguaje verbal, no verbal y paraverbal de los participantes para su posterior análisis. Se hizo énfasis en los discursos más controvertidos y en las discusiones más extensas sostenidas por los adolescentes. A partir de los datos recolectados en los talleres, se definieron las dimensiones y las categorías analíticas operativas a desarrollar en el presente artículo. En un principio se tuvo en cuenta ciertas categorías a priori que el equipo consideró oportuno trabajar. En este sentido, en base a una amplía revisión de literatura científica internacional específica en este campo, se seleccionaron algunos conceptos a abordar en las actividades por tratarse de temas de alta prevalencia en población adolescente, por ejemplo: sexting, cyberbullying y grooming. A partir de los discursos generados en el abordaje de estos conceptos o categorías se definieron las siguientes dimensiones emergentes y relevantes para el análisis del artículo.

\section{Resultados y discusión}

El análisis de la información recabada se realiza en tres apartados que engloban los resultados más destacados de la experiencia. Los mismos son: 1) la emergencia de nuevas prácticas sexuales y la resignificación del concepto de sexualidad; 2) las redes sociales online como dispositivos de control y su vinculación con la sexualidad; 3) noción de riesgo y prácticas sexuales virtuales seguras en las redes sociales online. Se destaca que el análisis cualitativo-interpretativo se basa en los discursos de los participantes en función de las técnicas aplicadas. Se considera que la selección de estos apartados es la más oportuna porque permiten abrir el debate en torno a las nuevas modalidades de ejercicio activo de la sexualidad de los adolescentes mediatizada por las TIC, también se discuten los sentidos y significados contemporáneos que tiene este grupo social sobre la sexualidad, la función de control social de las redes sociales online en el marco de los procesos de normalización que se producen en los entornos digitales, así como también, 
se presenta una discusión actual sobre el impacto potencial de algunas prácticas sexuales virtuales no seguras, a la vez que se ahonda en las medidas de seguridad mayormente implementadas por los y las adolescentes.

\subsection{La emergencia de nuevas prácticas sexuales y la resignificación del concepto de sexualidad}

El concepto de sexualidad está en constante construcción y transformación, las diferentes expresiones de la sexualidad son dinámicas. Ésta siempre está atravesada por una o varias motivaciones, generalmente vinculadas a uno de los dos fines de la sexualidad: el erótico placentero y/o el reproductivo (Ramos, Forrisi y Gelpi, 2016). En las últimas décadas, cada vez más, las prácticas se orientan mayormente al fin erótico/placentero y se produjo el fin de la clandestinidad del erotismo y esto es atribuible en parte a la difusión masiva de la pornografía (Bozón, 2004).

Novedosos objetos como internet, las redes sociales online y los smartphones posibilitan la existencia de nuevos sujetos y prácticas, inclusive sexuales. Hasta hace algunas décadas, era impensable que la sexualidad se expandiera al ámbito digital, en la actualidad, distintos espacios virtuales como son las aplicaciones para conocer gente y las redes sociales online han alterado la normatividad de las conductas sexuales, provocando que las representaciones culturales de la sexualidad se hayan ampliado (Bozón, 2004; Sibilia, 2015; Ballester et al., 2018). Estas posibilidades de intercambio entre los sujetos habilitan nuevas fantasías, deseos y eróticas. Los significados y sentidos que cobran estas prácticas son singulares y están influenciados por el momento del ciclo de vida en el que se encuentren las personas.

Los escenarios sexuales entendidos desde la perspectiva de Bozón (2004) como guías de orientación o de lectura que permiten a los sujetos situarse y atribuirle un sentido sexual a las sensaciones, situaciones, palabras y estados corporales se han ampliado y en los intercambios en las redes sociales online se produce sexualidad, deseo y, por ende, prácticas sexuales online. Sin embargo, no siempre se acaban materializando en un encuentro sexual en la realidad material concreta.

Los adolescentes de todos los contextos socioeconómicos que participaron de los talleres afirman que conocen personas en las redes sociales online con un objetivo afectivo-sexual, aunque existen expectativas diferenciales según el sexo. Este punto también está atravesado por el género. Mientras en los discursos de los varones, la principal motivación es predominantemente sexual, en las mujeres predomina la 
dimensión afectiva. Hubo relatos que hicieron referencia a situaciones donde luego de concretado el encuentro sexual, generalmente, los varones, unilateralmente decidían interrumpir el vínculo con su partenaire.

Luego de que nos vimos y estuvimos, llegué a mi casa, abri WhatsApp para hablarle y me había bloqueado, me sentí usada (adolescente mujer)

Hablamos un par de días, estuvimos y después nunca más, si te ves varias veces se enamoran y no te la sacás más de encima (adolescente varón)

Desde la perspectiva de los participantes, este aspecto, era productor de malestar psíquico, especialmente en las mujeres. Al parecer, existe una tensión intersubjetiva entre las expectativas diferenciales afectivo-sexuales, las cuales, permiten ser analizadas desde la perspectiva de género.

A su vez, la práctica del sexting forma parte de la vida cotidiana, está naturalizada y es popular entre los diferentes grupos de adolescentes. Los participantes sostienen que la herramienta predilecta para el intercambio de imágenes y/o videos con fines eróticos, excitatorios y/o masturbatorios son Snapchat y WhatsApp. La última aplicación mencionada es la más utilizada en la población uruguaya, por lo que, es esperable que sea en ella donde más se produce la práctica del sexting (Grupo Radar, 2016). Los adolescentes también identificaron a Snapchat por las facilidades que otorga para el intercambio de este tipo de materiales y por las garantías de seguridad que ofrece.

Snapchat es la mejor, se destruye la foto o el video, me deja tranquila (adolescente mujer)

Si tenés confianza la cosa es por WhatsApp, pero si recién estás hablando es mejor Snapchat (adolescente varón)

Igualmente, al parecer, el concepto sexting no ha permeado en la realidad local y para hacer referencia a los intercambios sexuales virtuales, los adolescentes utilizan la palabra de origen anglosajón pack. Además, consideran que el sexting de ningún modo configura una iniciación sexual para quienes aún no se han iniciado. Hay un grupo mayoritario que cree que, en caso de estar en pareja, dicha práctica no se interpreta como un engaño, quitándole trascendencia desde sus perspectivas a las acciones y conductas vinculadas a la sexualidad en entornos digitales.

Aunque tanto varones como mujeres practican el sexting, generalmente, los varones son quienes solicitan en mayor medida fotografías, videos y/o videollamadas e incluso en ocasiones envían material explícito sin consentimiento, lo que configura situaciones de violencia sexual. Esta realidad condice con el estudio coordinado por 
Burén y Lunde (2018). Estas características podrían vincularse con la construcción social histórica de la sexualidad de los varones y mujeres y, con los mandatos vinculados a la masculinidad, especialmente, los relativos al ejercicio de la sexualidad y a la demostración de virilidad.

A veces te mandan fotos o videos de la nada, estás cenando, tranqui, abrís un mensaje y te encontrás con un pene, es desagradable (adolescente mujer) Yo no quería mandarle nada, lo hice porque me gustaba, no quería que me dejara de hablar (adolescente mujer)

En las mujeres, el deseo, a veces queda en segundo plano y ceden a las presiones de sus parejas o partenaires que están conociendo por temor a que les ignoren, siendo las principales víctimas de sextorsion y sexrevenge. Los varones son quienes más viralizan este tipo de material y en muchos casos lo hacen dentro de su propio grupo de pares, mayoritariamente conformado por varones. Esto se relaciona con los resultados de la investigación de Burén y Lunde (2018), donde encontraron que las mujeres perciben tener más experiencias negativas en torno a esta práctica y estar más expuestas a situaciones de violencia gracias a la difusión de material con contenido de ellas. En varias instancias de taller se mencionó que tenían conocimiento sobre casos cercanos de viralización de fotos y/o videos, donde las víctimas eran mujeres. Las adolescentes mujeres manifestaron que los varones clasifican los pack que reciben de acuerdo a si cumplen con los parámetros estéticos hegemónicos, reforzándose en las TIC la cosificación del cuerpo de las mujeres dentro de una competencia simbólicamente masculina.

Un amigo del liceo me contó que tienen un grupo en WhatsApp donde comparten nuestros packs, opinan y nos ponen nota, me pareció un asco, quedé re indignada (adolescente mujer)

También se hizo énfasis en que el escenario de viralización frecuente era luego de una ruptura de noviazgo o de un vínculo afectivo-sexual, donde como castigo, se difundía ese material. Esto permite pensar que en ocasiones, las redes sociales online ofician como un dispositivo de control en los vínculos afectivo-sexuales de los adolescentes. En este sentido, las relaciones de género y las estructuras de poder impactan en el ejercicio de la sexualidad inclusive en las redes sociales online. Las prácticas sexuales facilitadas por las TIC refuerzan las relaciones de género y las estructuras de poder. A partir de los intercambios producidos pareciera que, en la virtualidad, se mantiene la asimetría en los roles sexuales a la hora del ejercicio de 
la sexualidad y las principales consecuencias negativas de las distintas prácticas recaen sobre las mujeres, siendo posible identificar a las redes sociales online como otro espacio donde se producen y reproducen desigualdades de género.

\subsection{Las redes sociales online como dispositivos de control y su vinculación con la sexualidad}

Las redes sociales online se han transformado en la modernidad tardía en el panóptico descripto por Foucault (2002). Más allá de los aspectos positivos de dichas redes, ellas también se caracterizan por su carácter potencial de vigilancia, control y castigo. Salvo WhatsApp que le permite a los usuarios tener un mayor control, en las redes sociales online, las personas constantemente son visibles (estado de conexión, última hora de conexión, geolocalización, cantidad de amigos, amigos en común), buscan la aprobación y el reconocimiento del entorno mediante distintas acciones (estados, fotos, videos y transmisiones en vivo, entre otras funciones disponibles). En esta lógica de funcionamiento actual, los cuerpos sexuados cobran vital importancia por lo que representan (especialmente durante la adolescencia) y sobre ellos se ejerce el juicio, aprobación y sanción de acuerdo con lo deseado y considerado "bello" en una sociedad, en una cultura determinada y en un contexto-socio histórico dado. Incluso, se deben de considerar los atravesamientos relativos al sexo, la clase social, la edad, el origen étnico-racial, la orientación sexual, la identidad de género y la identidad sexual, entre otras. Las redes sociales online configuran un nuevo espacio normalizador basado en modelos hegemónicos con relación al género y la sexualidad, que condicionan los comportamientos e interacciones de los sujetos en las redes más allá de la orientación sexual de estos (Sibilia, 2018b).

Desde la perspectiva de los adolescentes, quienes producen contenidos son quienes se ajustan a las exigencias estéticas y quienes no, son meros consumidores (Castells, 2000). Los mensajes e imágenes que circulan por las redes sociales online son interpretados de distintos modos, afectan la configuración del deseo, la producción de subjetividad y la identidad de los adolescentes. También impactan en la socialización de género y sexual de este grupo social y reeditan dinámicas de discriminación y exclusión social hacia ciertos sujetos, cuerpos e identidades.

Por lo general las que pasan subiendo fotos al Instagram son los que están buenas, las feas ni foto de perfil tienen, solo comparten memes en Facebook o cosas frikis (adolescente varón). 


\subsection{Noción de riesgo y prácticas sexuales virtuales seguras en las redes sociales online}

Un mayor acceso y uso de las distintas herramientas digitales incrementa la posibilidad de enfrentarse a un riesgo. La gestión de riesgos por parte de los usuarios se relaciona directamente con la reducción de daños potenciales (Livingstone, Mascheroni y Staksrud, 2015). Existe una brecha generacional entre lo que es considerado de riesgo para los adultos y los adolescentes. Con relación a los riesgos en las redes sociales online, los participantes informan que las familias y otros agentes sociales no cuentan con tanta información sobre las potenciales consecuencias negativas de algunas prácticas en entornos digitales, pero entre pares si se habla de estas prácticas. Los adolescentes no siempre cuentan con un referente adulto que los acompañe en el proceso de aprendizaje de los riesgos existentes en entornos digitales, especialmente por dos motivos: estas novedosas formas de interacción a veces le son distantes a los adultos y por un tema generacional, algunos de ellos son considerados analfabetos digitales (Livingstone y Third, 2017).

Mis padres no tienen idea de que es un pack o las cosas que se hacen en Snapchat (adolescente mujer)

74 En sociedades donde la extimidad ha permeado los distintos grupos sociales, desde la perspectiva de los adolescentes, la obtención de reconocimiento -en tanto recompensa-supera a la evaluación de los riesgos inmediatos y sus posibles consecuencias. Aunque para los participantes la mayoría de intercambios sexuales virtuales no conllevan consecuencias negativas, cuando suceden situaciones que tienen un desenlace no deseado, las víctimas, dependiendo la singularidad, presentan diversos efectos bio-psico-sociales. Existen datos recientes de un estudio realizado en Uruguay que sostiene que, las mujeres se sienten más inseguras en entornos digitales que sus pares varones (UCU y UNICEF, 2018).

A partir del intercambio, se concluye que, los recaudos más usados por los adolescentes para practicar el sexting de un modo seguro son: usar predominantemente Snapchat y WhatsApp por las seguridades que ofrecen, eliminar el material enviado y/o recibido, no enviar fotos y/o videos donde se pueda reconocer sus rostros, cicatrices y/o tatuajes, aplicar la pantalla de bloqueo y tener contraseñas en sus teléfonos móviles para que terceros no tengan acceso a secciones como la galería, entre otras. En ocasiones, argumentaron que ni siquiera teniendo precauciones se evitan situaciones de vulneración de derechos y conocen varios casos donde alguna de las partes muestra la conversación personalmente en la propia institución edu- 
cativa y sin la necesidad de viralizar las fotos y/o videos se producen consecuencias negativas. A su vez, en todos los talleres manifestaron desconocer a la división que se encarga de este tipo de delitos a la interna del Ministerio del Interior.

Los participantes también informaron sobre la existencia de cuentas no oficiales de las distintas instituciones educativas en las redes sociales online Facebook y Twitter, donde los adolescentes, desde el anonimato, postean los packs de otros estudiantes de las instituciones educativas. Antes del presente ciclo de talleres, la mayoría de los participantes no se habían detenido a problematizar sobre esta práctica cotidiana y sus efectos, ni tampoco habían conseguido asociar esta vulneración de derechos a otros problemas de salud mental que habían presentado las personas victimizadas, por ejemplo, dentro de los más visibles y graves: cuadros de depresión, ansiedad e intentos de autoeliminación.

Generalmente, coincidían en que la mayoría de los packs posteados eran de mujeres que dependiendo del cuerpo obtenían mayor popularidad entre los varones -en tanto objeto de deseo- y más rechazo social por parte de las mujeres -en tanto vista como una "puta"-. A su vez, para los varones, los casos donde se ponía en duda la virilidad era cuando sus penes no alcanzaban el tamaño estándar. Estos elementos coinciden con los límites de género descriptos por Guasch (2007) cuando afirma que el límite de género en las mujeres es devenir en puta y en los varones ser homosexual con la correspondiente pérdida de virilidad. Los estereotipos, mandatos y normas de género atraviesan las prácticas sexuales virtuales y en ocasiones tienen destinos diferenciados dependiendo del sexo de la persona.

La otra vez se viralizó la foto de un compa que la tenía re chica y lo descansamos todo el año (adolescente varón)

Después que todo el mundo vio la foto en bolas de Andrea nadie quería estar con ella, era la puta del liceo (adolescente mujer)

A su vez, en las discusiones, se observó que pareciera existir una brecha diferencial de género en cuanto a la interiorización de la noción de riesgo y determinadas prácticas online seguras. Las mujeres enumeraron más estrategias de seguridad y una mayor solidaridad de género que sus pares varones, mientras que, estos últimos, mostraron elementos asociados a una mayor impulsividad, lo cual, podría llegar a ser abordado desde los aportes de los estudios de las masculinidades.

A mi si me hablan y me dicen un par de cosas que me calientan me animo a todo de una (adolescente varón) 
Entre nosotras nos hablamos, nos pasamos los nombres de los que le piden a todas, como para que una de nosotras no caiga en eso porque te hace sentir importante, lo único que quieren es un pack (adolescente mujer)

Todo lo antes expuesto se relaciona con el concepto de ciudadanía digital. Dicho concepto remite a derechos y obligaciones de los ciudadanos en sus interacciones y prácticas a través de las TIC. La ciudadanía digital coopera para la comprensión de temas sociales, culturales y políticos que se expresan en las TIC, así como a la aplicación de conductas pertinentes a esa comprensión ajustándose a los principios de ética, legalidad, seguridad y responsabilidad en el uso de internet, redes sociales online y otras tecnologías disponibles (Robles, 2009). En ese sentido, una de sus vertientes se vincula con la educación porque alude a la formación en el uso de las TIC para hacer un uso responsable y seguro de las mismas, disminuyendo así la exposición potencial a riesgos en entornos digitales. En ese sentido, a partir de este ciclo de talleres, es posible concluir que se debe fomentar en nuestra realidad local, la construcción de ciudadanía digital en población adolescente debido al uso arbitrario que hacen de las TIC en general y de las redes sociales online en particular.

\section{Consideraciones finales}

La experiencia se evalúa positivamente, hubo cooperación entre los distintos actores e instituciones implicadas en el proceso. Se construyeron nuevas alianzas interinstitucionales, se coordinó con actores de la comunidad educativa, se trabajó con las demandas planteadas por parte de las instituciones y se cumplió con la totalidad de los objetivos previstos en cada instancia taller con los adolescentes de cada centro educativo.

Las instituciones que adhirieron a la propuesta de talleres se caracterizaron por reconocer la alta prevalencia de casos de sexting en la población adolescente. También emergieron como otra constante mencionada por las instituciones, los efectos de esta práctica en el cotidiano escolar y la falta de herramientas con las que cuentan los actores educativos para dar respuestas sólidas y eficaces a estos nuevos fenómenos que siembran incertidumbre y preocupación en el mundo adulto.

A partir del intercambio con los adolescentes se concluye que, internet, las redes sociales online y los smartphones posibilitan la existencia de nuevos sujetos y prácticas, inclusive sexuales. Las redes sociales online y otros espacios virtuales han producido cambios en la normatividad de las conductas sexuales, ampliando las representaciones culturales de sexualidad. Los significados y sentidos que cobran 
estas prácticas son singulares y se ven influenciadas por diferentes aspectos, como por ejemplo, aspectos socioculturales como el sistema sexo-género, historia de vida del sujeto, características de personalidad, entre otros. Igualmente, se debe destacar que las prácticas sexuales virtuales no necesariamente se acaban materializando en un encuentro sexual en la realidad material concreta.

La práctica del sexting forma parte de la vida cotidiana, está naturalizada y es popular en los diferentes grupos de adolescentes. Las herramientas predilectas para el intercambio de imágenes y videos con fines eróticos, excitatorios y masturbatorios son Snapchat y WhatsApp. Sin embargo, el concepto de sexting no ha permeado en la realidad local y para hacer referencia a los intercambios sexuales virtuales, los adolescentes utilizan la palabra de origen anglosajón pack.

Asimismo, los adolescentes de todos los contextos socioeconómicos que participaron del ciclo de talleres afirman que han conocido personas en las redes sociales online con un objetivo afectivo-sexual. Dichos objetivos varían dependiendo mayoritariamente del sexo de la persona. En este sentido, desde una perspectiva de género, es posible concluir que, en la virtualidad se mantiene la asimetría en los roles sexuales a la hora del ejercicio de la sexualidad y las principales consecuencias negativas de las distintas prácticas recaen sobre las mujeres, siendo posible identificar a las redes sociales online como otro espacio donde se producen y reproducen desigualdades de género.

En general, los mensajes e imágenes que circulan por las redes sociales online son interpretados de distintos modos, afectan la configuración del deseo, la subjetividad y la identidad de los adolescentes. También impactan en la socialización de género y sexual de este grupo social y reeditan dinámicas de discriminación y/o exclusión social hacia ciertos sujetos, cuerpos e identidades.

Por lo antes expuesto, sería importante que se continúe fomentando la construcción de ciudadanía digital en los espacios formales de educación para que la población adolescente desarrolle herramientas para hacer un uso más responsable y seguro de las TIC.

\section{Referencias bibliográficas}

Balaguer, R. (2008). ¿Adicción a internet o adicción a la existencia? Revista Digital Universitaria, 9(8), 1067-6079. 
Ballester, R.; Castro, J.; Ruiz, E.; Gil, B. y Giménez, C. (2018). Sexo, mentiras e internet. International Journal of Developmental and Educational Psychology, 1(1), 43-52.

Bernete, F. (2009). Usos de las TIC, Relaciones sociales y cambios en la socialización de las y los jóvenes. Revista Latinoamericana de Comunicación, 3(88), 97-114.

Bozon, M. (2004). Sociología da sexualidade. Rio de Janeiro: Editora FVG.

Burén, J. y Lunde, C. (2018). Sexting Among Adolescents: A Nuanced and Gendered. Online Challenge for Young People. Computers in Human Behavior, 85, 210-217.

Castells, M. (2000). La era de la información. Volumen III: El poder de la identidad. Ciudad de México: Siglo XXI Editores.

Cornejo, M. y Tapia, M. L. (2011). Redes sociales y relaciones interpersonales en internet. Fundamentos en Humanidades, 22(24), 219-229.

Dean, M. (1994). A social structure of many souls: moral regulation, government, and selfformation. Canadian Journal of Sociology / Cahiers canadiens de sociologie, 19(2), 145-168.

Del Hoyo, M., Fernández, C. y García, M. C. (2014). Jóvenes comprometidos en la Red: El papel de las redes sociales en la participación social activa. Comunicar, 22(43), 35-43.

Fernández, A.M. (2015). Com-posiciones actuales de las identidades sexuales. Revista Generaciones, 4(4), 1-18.

Fernández Sánchez, N. (2013). Trastornos de conducta y redes sociales en internet. Salud Mental, 36(6), 521-527.

Foucault, M. (2002). Vigilar y castigar: nacimiento de la prisión. Buenos Aires: Siglo XXI Editores.

Fox, N. (1998). Foucault, foucauldians and sociology. The British Journal of Sociology, 49(3), 415-433.

Giddens, A. (1992). La transformación de la intimidad. Sexualidad, amor y erotismo en las sociedades modernas. Madrid: Ediciones Cátedra.

Grupo Radar. (2016). El perfil del internauta uruguayo 2016. Resumen Ejecutivo. Montevideo: Grupo Radar.

Guasch, O. (2007). La crisis de la heterosexualidad. Barcelona: Editorial LAERTES.

Kendall, L. (2000). Oh No! I'm a Nerd! Hegemonic masculinity on an online forum. Gender and Society, 14(2), 256-274.

Klein, A. (2004). Adolescencia: Un puzle sin modelo para armar. Montevideo: Ed. Psicolibros.

Linne, J. (2018). Nomadización, ciudadanía digital y autonomía. Tendencias juveniles a principios del siglo XXI. Chasqui. Revista Latinoamericana de Comunicación, 137, 37-52. 
Livingstone, S. y A. Third. (2017). Children and young people's rights in the digital age: An emerging agenda. New Media \& Society, 19(5), 657-670.

Livingstone, S., G. Mascheroni y E. Staksrud. (2015). Developing a frameworkfor researching childrens online risks and opportunities in Europe. Londres: EU Kids Online.

López Gómez, A. (Coord.). (2015). Adolescentes y sexualidad. Investigación, acciones y política pública en Uruguay (2005-2014). Montevideo: UNFPA.

Louro, G. (2000). Pedagogias da sexualidade. O corpo educado: Pedagogias da sexualidade. Belo Horizonte: Autêntica.

Márquez, I. (2017). El smartphone como metamedio. Observatorio Journal, 11(2), 61-71.

Mercado C., Pedraza, F. y Martínez, K. (2016). Sexting: su definición, factores de riesgo y consecuencias. Revista sobre la infancia y la adolescencia, 10, 1-18.

Ministerio de Desarrollo Social e Instituto Nacional de la Juventud y Adolescencia (MIDES y INJU). (2015). Informe. Tercera Encuesta Nacional de Adolescentes y Juventud. Montevideo: MIDES.

Narvaja, M. E. y De Piero, J. L. (2016). Practicas juveniles extimas. Sexting y vlogging. Aposta. Revista de Ciencias Sociales, 69, 239-270.

Noboa, L. (2010). Tendencias de acceso y usos de Tics en Uruguay. Montevideo: PISA Uruguay.

Pérez, P., Flores, J., de la Fuente, S., Álvarez, E., García, L., y Gutiérrez, C., (2011). Guía sobre adolescencia y sexting: qué es y cómo prevenirlo. Madrid: Observatorio de la Seguridad de la Información de INTECO y Pantallas Amigas.

Ramos, V; Forrisi, F. y Gelpi, G. (2015). Nociones básicas sobre sexualidad, género y diversidad: Un lenguaje en común. En P. López, F. Forrisiy G. Gelpi (Coord.), Salud y diversidad sexual. Guía para profesionales de la salud. Montevideo: MSP.

Ramos, V. (2015). Consideraciones conceptuales: Adolescencia, sexualidad y derechos. En A. López (Coord.), Adolescentes y sexualidad. Investigación, acciones y política pública en Uruguay (2005 - 2014). Montevideo: UNFPA.

Regalado, A. B, Fajardo, M. I., Casanueva, N., Bermejo, M. L y Castro, F. (2012). ¿Para qué utilizan las adolescentes las redes sociales? International Journal of Developmental and Educational Psychology, 1(1), 609-628.

Robles, J. M. (2009). Ciudadanía digital. Una introducción a un nuevo concepto de ciudadano. Barcelona: Editorial UOC.

Sabater, C. (2014). La vida privada en la sociedad digital. La exposición pública de los jóvenes en internet. Aposta. Revista de Ciencias Sociales, 61, 1-32.

Sibilia, P. (2018a). Você é o que Google diz que você é: a vida editável, entre controle e espetáculo. Intexto, 42, 214-231. 
Sibilia, P. (2018b). Los cuerpos visibles en la contemporaneidad: De la purificación mediática a la explicitud artística. Revista Kaypunku de Estudios Interdisciplinarios de Arte y Cultura, 4(1), 175-197.

Sibilia, P. (2015). Mal de amores. Afectos y vínculos eróticos en tiempos hiperconectados. Revista de la Asociación Argentina de Psicología y Psicoterapia de Grupo, 38, 83-90.

Sibilia, P. (2009). En busca del aura perdida: Espectacularizar la intimidad para ser alguien. Psicoperspectivas. Individuo y Sociedad, 8(2), 309-329.

Sibilia, P. (2008). La intimidad como espectáculo. Buenos Aires: Fondo de Cultura Económico.

Smith, M. (1992). Postmodernism, urban ethnography, and the new social space of ethnic identity. Theory and Society, 21(4), 493-531.

Somers, M. (1994). The narrative constitution of identity: a relational and network approach. Theory and Society, 23(5), 605-649.

Stryker, S. y Burke, P. (2000). The past, present, and future of an identity theory. Social Psychology Quarterly, 63(4), 284-297.

Topa, G. (2010). Presentación del monográfico: Psicología e internet. Acción Psicológica, 7(1), 5-6.

Rudolph, S. (2003). Engaging subjective knowledge: how Amar Singh's diary narratives of and by the self explain identity formation perspectivas. PoliT/C, 1 (4), 681-694.

Turkle. S. (1999). Identity and cyberspace. Contemporary Sociology, 28(6), 643-648.

Turkle, S. (1984). The second self: computers and the human spirit. New York: Simon \& Schuster.

Universidad Católica del Uruguay y Fondo de las Naciones Unidas para la Infancia (UCU y UNICEF). (2018). Informe Kids Online Uruguay. Niños, niñas y adolescentes conectados. Montevideo: Mastergraf.

Velázquez, M; López, S. y Arellano, A. (2013). Sexting: La sexualidad responsable también debe ejercerse en las redes sociales. Santiago de Chile: XXIX Congreso Latinoamericano de Sociología.

Viñar, M. (2009). Mundos adolescentes y vértigo civilizatorio. Montevideo: Ediciones Trilce.

Warren, M. (1990). Ideology and the Self. Theory and Society, 19(5), 599-634. 